\title{
Protective effects of Quercetin and chronic moderate exercise (training) against oxidative stress in the liver tissue of streptozotocin-induced diabetic rats
}

\author{
IC Chiş ${ }^{1}$, A Mureşan' ${ }^{1}$ A Oros ${ }^{2}$, AL Nagy², S Clichici ${ }^{1}$ \\ ${ }^{1}$ Department of Physiology, "Iuliu Hatieganu” University of Medicine and Pharmacy, \\ Cluj-Napoca, Romania \\ ${ }^{2}$ Department of Veterinary Toxicology, University of Agricultural Sciences and Veterinary Medicine, \\ Cluj-Napoca, Romania
}

Received: August 4, 2014

Accepted: December 10, 2015

\begin{abstract}
Background: To investigate the protective effects of Quercetin administration associated with chronic moderate exercise (training) on oxidative stress in the liver in streptozotocin-induced diabetic rats. Methods: Diabetic rats that performed exercise training were subjected to a swimming training program (1 hour/day, 5 days/week, 4 weeks). The diabetic rats received natural antioxidant, Quercetin $(20 \mathrm{mg} / \mathrm{kg}$ body weight/day) for 4 weeks. At the end of the study, all animals were sacrificed and liver samples were collected for estimation: some oxidative stress markers (malondialdehyde, MDA and protein carbonyls groups, PC), the activity of antioxidant enzymes (superoxide dismutase, SOD and catalase, CAT), reduced glutathione (GSH) level and reduced (GSH) and oxidized (GSSG) glutathione ratio. Results: Diabetic rats submitted to exercise training showed significantly increased the oxidative stress markers (MDA and PC) and a reduction of antioxidant enzyme (SOD and CAT) activity, GSH level and GSH/ GSSG ratio in hepatic tissues. A decrease in the levels of oxidative stress markers associated with elevated activity of antioxidant enzymes, the GSH level and GSH/GSSG ratio in the hepatic tissue were observed in Quercetin-treated diabetic trained rats. Conclusions: These findings suggest that Quercetin administration in association with chronic moderate exercise exerts a protective effect in diabetes by attenuating hyperglycemia-mediated oxidative stress in hepatic tissue.
\end{abstract}

Keywords: diabetes mellitus, liver, Quercetin, chronic moderate exercise, oxidative stress

Diabetes mellitus (DM) is a chronic endocrine-metabolic disorder characterized by hyperglycemia and lipid, protein and carbohydrate metabolic abnormalities, which determine an increase in the risk of complications from vascular diseases in the long run $(10,17)$. The number of individuals affected by DM is increasing all over the world because of physical inactivity, increased prevalence of obesity, urbanization and population aging. Hyperglycemia is associated with the production of reactive oxygen species (ROS) and with the lowering of antioxidant capacity, which play an important role in the oxidative damage especially in the liver, kidneys, eyes, nerves, pancreas and blood vessels $(2,5,7,24,37)$.

Oxidative stress plays an important role in the pathogenesis and progression of DM and in the diabetic complications. The implication of oxidative stress in insulin resistance and in the pathogenesis of DM is explained not only through the generation of reactive oxygen and

Corresponding author: Irina Camelia Chis $\mathrm{MD}, \mathrm{PhD}$

Department of Physiology, "Iuliu Hatieganu" University of Medicine and Pharmacy

1-3, Clinicilor Street, RO 400023, Cluj-Napoca, Romania.

Phone: +40-744-707080; Fax +40-264-597257; E-mail: irinnaus@yahoo.com 
nitrogen species (RONS) but also through the alteration of endogenous antioxidant enzymes. Hyperglycemia generates RONS through multiple pathways such as: the activation of nuclear factor (NF)- $\kappa \mathrm{B}$ which increases the expression of inducible nitric oxide synthase (iNOS) and the increased generation of nitric oxide (9); increased mitochondrial production of the superoxide anion radicals (37); glucose autoxidation (31); non-enzymatic glycation of proteins $(25,26)$; the activation of protein kinase $C$ (18); etc. Nitric oxide reacts with the superoxide and forms peroxynitrite, which is a strong oxidant that increases lipid peroxidation, protein nitration and LDL oxidation. These modifications are responsible for insulin resistance and diabetes tissue injury (11).

Many recent studies presented the effects of administering natural antioxidants on DM and in preventing the progression of diabetic complications, but the results are still incompletely comprehended $(7,14,19)$. A highly promising candidate for the prevention of adverse effects of oxidative stress in DM is Quercetin (3,4,5,7,3,4-penta-hydroxyflavone), which is one of the most abundant flavonoids in the human diet, as it is present in fruits and vegetables such as onions, citrus fruits, berries, red grapes, red wine, broccoli, bark roots, flowers, and tea (6). Quercetin has protective effects against beta-cell damage and it's capable of decreasing blood glucose levels in diabetes $(1,2)$. Recent studies demonstrated that Quercetin has strong antioxidant and protective effects against oxidative stress in streptozotocin (STZ)-induced diabetic rats, protecting various tissues and organs $(4,10,19)$ such as the liver (11), pancreatic gland, kidneys (2) and testis (22).

Another important role in the diabetes therapy is that of exercise training. The exercise training has been reported to be effective in the prevention and delay of the diabetes onset, increase of insulin sensitivity and improvement of the glucose metabolism $(35,38)$. Recent experimental research has reported therapeutic preventive and protective effects of exercise training on diabetes by decreasing the oxidative stress and preserving the pancreatic $\beta$-cell integrity (9). Exercise training has also been reported as being able to increase the sensitivity to insulin of the skeletal muscles, to improve insulin resistance and regulate glucose homeostasis in models with type 2 diabetes (23). However, the mechanism remains poorly understood.

The experimental induction of diabetes with STZ is very commonly used in rats. Streptozotocin is an antibiotic produced by Streptomyces achromogenes and is often used as a result of its toxic effects on pancreatic $\beta$-cells. Its cytotoxic actions in the pancreas are associated with the generation of ROS causing oxidative damage and vascular dysfunction $(30,34)$.

In the present study we evaluated the effects of Quercetin associated with chronic moderate exercise (training) on fasting blood glucose level and on lipid peroxidation, protein carbonylation, enzymatic and non-enzymatic antioxidants in the liver of hyperglycemiamediated oxidative stress in STZ-induced type 1 diabetic rats. In addition, the effects of Quercetin associated with chronic moderate exercise (training) on the histopathological alterations of the liver were also studied in control and diabetic rats.

\section{Materials and Methods}

\section{Drugs and chemicals}

Streptozotocin (STZ) and Quercetin (Que) were obtained from Sigma Chemical Company Inc., the UK. Streptozotocin was freshly dissolved in citrate sodium buffer $(0.01 \mathrm{M}, \mathrm{pH} 4.5)$ and maintained on ice prior to use. The Que was suspended in $0.5 \%$ carboxymethylcellulose (CMC) solution as a vehicle. 


\section{Animals}

Eighty-eight healthy Wistar albino male rats (three months old) were used in this study. The animals were divided into eight experimental groups. The first four groups included 10 rats each, whereas the last four groups were made up of 12 rats each. Given the diabetic rat mortality, the final statistical analysis was carried out in 10 rats from each experimental group. The rats were purchased from the Experimental Animal House of the Faculty of Medicine of "Iuliu Hatieganu" University of Medicine and Pharmacy of Cluj-Napoca, Romania. The rats' mean weight was 250-300 grams (g) at the start of the trials. All the animals used in the experiment were kept for ten days to acclimatize to the conditions of the Animal House Laboratory at the Physiology Department before being introduced in the study. Throughout the entire period of the experiment, all the rats were maintained in special cages, which were kept under control, artificially illuminated (12 h dark/12 h light cycle), at a temperature of $21-23^{\circ} \mathrm{C}$ and at $50-60 \%$ humidity in an animal room. The animals were given standard rat pellets diet and water ad libitum. All the experiments were performed according to the approved animal protocols of the Ethical Committee on Animal Welfare of the "Iuliu Hatieganu" University in accordance with the Romanian Ministry of Health and complying with Guidelines in the Use of Animals in Toxicology.

\section{Induction of experimental diabetes mellitus by STZ}

The study was carried out on an animal model of type 1 diabetes induced with STZ. Diabetes was induced in rats by a single intraperitoneal (i.p.) injection of STZ (40 mg/kg body weight) given to overnight fasting rats $(7,21)$. Animals of the control groups were given only a single i.p. injection of citrate sodium buffer at the same volume used to dissolve STZ. Ninety-six hours after STZ administration, DM was confirmed by measuring the fasting blood glucose (FBG) concentration. The blood was drawn from the retro-orbital venous plexus of overnight fasting animals into tubes containing potassium oxalate and sodium fluoride as anticoagulant, whereas the plasma glucose was estimated using a commercial glucose kit (Qualigens Diagnostics - Accu-Chek-ROCHE). Rats that had a higher FBG level than $250 \mathrm{mg} / \mathrm{dL}$ (13.89 $\mathrm{mmol} / \mathrm{L}$ ) were included in the study as diabetic rats $(7,27)$. One week after STZ administration, the FBG level was measured and the Que administration and exercise training started.

\section{Chronic Moderate Exercise (training) protocol}

Each day, animals were transported to an experimental room where exercise animals were forced to swim. Three rats from each exercise group were placed in a cylindrical tank with a diameter and height of 60 and $100 \mathrm{~cm}$, respectively, in water at a depth of 30-45 cm. To minimize stress associated with cold or hot water exposure, water temperature was monitored and maintained at $36^{\circ} \mathrm{C}$. The rats were trained to swim for over one week, for $15 \mathrm{~min} /$ day ( 5 days/week); the exercise protocol was gradually increased by $15 \mathrm{~min} /$ day until a swimming period of 1 hour/day ( 1 week) was attained in order to reduce water-induced stress. After the initial training, the rats underwent chronic moderate exercise for 1 hour/day, 5 days/week, for 4 weeks. The animals were forced to swim until they became exhausted and remained at the water surface. The exercise program was conducted essentially as described by Teixeira de Lemos et al. (35) with some modifications, though. The sedentary rats were subjected to the same sampling and handling procedures as the exercise training animals, but they remained in their cages without food and water for the duration of the swimming exercise period. 


\section{Experimental design}

The animals were divided into eight experimental groups: the $1^{\text {st }}$ Group (control + sedentary, CS)- non-diabetic, sedentary untreated control rats; the $2^{\text {nd }}$ Group (control + exercise, CE)non-diabetic, trained untreated control rats; the $3^{\text {rd }}$ Group (control + sedentary + Quercetin, CSQ)- non-diabetic, sedentary control rats treated with Quercetin; the $4^{\text {th }}$ Group (control + exercise + Quercetin, CEQ)- non-diabetic, trained control rats treated with Quercetin; the $5^{\text {th }}$ Group (diabetes + sedentary, DS)- diabetic, sedentary untreated control rats; the $6^{\text {th }}$ Group (diabetes + exercise, DE)- diabetic, trained untreated control rats; the $7^{\text {th }}$ Group (diabetes + sedentary + Quercetin, DSQ)- diabetic, sedentary rats treated with Que; the $8^{\text {th }}$ Group (diabetes + exercise + Quercetin, DEQ)- diabetic, trained rats treated with Que.

The Que was orally administered via an intragastric tube $(0.6 \mathrm{ml} / \mathrm{rat})$, in a dose of $20 \mathrm{mg} /$ $\mathrm{kg}$ body weight once a day for 4 weeks. The rats from the groups: CS, CE, DS and DE were treated with CMC $(0.6 \mathrm{ml} / \mathrm{rat})$ administered orally once a day for 4 weeks. The Que and CMC administration in diabetic rats started one week after the STZ administration. The animals were then included in the long-term study of 35 days. For the entire duration of the experiment the following features were monitored in each animal: the water consumption (daily), food consumption (weekly), body weight (weekly), mortality (daily) and diuresis (daily). The FBG level was measured in all the experimental animals at the start of the experiment, after 96 hours and after 7 days of the STZ administration and at the end of the experiment. At the end of the final session of the training protocol, all animals were fasted for $12 \mathrm{~h}$, and then blood samples were collected from the retro-orbital venous plexus under diethyl ether anesthesia in order to measure the glycemia. After the blood samples were collected, the animals were anesthetized by intraperitoneal administration of sodium pentobarbital $(60 \mathrm{mg} /$ rat) and sacrificed by cervical dislocation. Also, samples of the liver of each animal were rapidly dissected and placed into ice-cold homogenization medium (HM). Parts of tissue were separated from connective tissue and were weighed, finely minced, and washed with HM.

\section{Preparation of biological samples}

The frozen liver tissue fragments were placed into plastic tubes and were gently homogenized $(20 \% \mathrm{w} / \mathrm{v})$ in the HM (220 mM mannitol, $70 \mathrm{mM}$ sucrose, $1 \mathrm{mM}$ EDTA, 0,1\% fatty acid-free albumin, $10 \mathrm{mM}$ Tris, $\mathrm{pH}$ 7.4) on ice using a Polytron homogenizer (Brinkman Kinematica Switezerland). The homogenates were centrifuged for $1 \mathrm{~min}$ at $500 \mathrm{rpm}$ and $0^{\circ} \mathrm{C}$. The supernatant was separated into separate tubes, stored at $-80^{\circ} \mathrm{C}$ and used for biochemical assays.

\section{Measurement of biochemical markers of oxidative stress}

Levels of oxidative stress in the hepatic tissue homogenate were indirectly measured through the measuring of the free radical production. The following biochemical markers of the effects of free radicals on lipid peroxidation and protein carbonylation were determined.

The lipid peroxidation was measured from the hepatic tissue homogenate by measuring the MDA levels (using the fluorimetric method with 2-thiobarbituric acid described by Conti method) (8). The MDA was spectrofluorimetrically determined in the organic phase using a synchronous technique with excitation at $534 \mathrm{~nm}$ and emission at $548 \mathrm{~nm}$. The MDA levels were expressed as nanomole per milligram protein (nmol MDA/mg protein).

Protein carbonylation was estimated from the hepatic tissue homogenate by measuring protein carbonyl (PC) group levels. The protein carbonyl derivates that are produced through the protein oxidative damage were determined using the fluorimetric method with 
2,4-dinitrophenyl-hydrazine (DNPH) (32). The readings were performed using a spectrophotometer at 355-390 $\mathrm{nm}$ and in order to calculate the remaining carbonyl fragments, the molar extinction coefficient with a value of $22,000 / \mathrm{M} / \mathrm{cm}$ was used. The carbonyl-derivative groups' levels were expressed as nanomole per milligram of protein (nmol/mg protein).

The activities of some antioxidant enzymes, superoxide dismutase (SOD) and catalase (CAT) and the non-enzymatic antioxidant (reduced glutathione) level and reduced and oxidized glutathione ratio in the hepatic tissue homogenate were assayed.

The SOD activity was assayed using the nitroblue tetrazolium (NBT) method proposed by Kakkar et al. (20). The NBT was reduced to blue formazen by superoxide, which has a strong absorbance at $560 \mathrm{~nm}$. One unit (U) of SOD is defined as the amount of protein that inhibits the rate of NBT reduction by $50 \%$ in 1 min under the assay conditions. The SOD activity was also expressed as units per milligram of protein (U/mg protein).

The CAT activity was assayed using the method proposed by Pippenger et al. (29). The method is based on recording the change in absorbance of a solution of $\mathrm{H}_{2} \mathrm{O}_{2} 10 \mathrm{mM}$ in potassium phosphate buffer $0.05 \mathrm{M}, \mathrm{pH}=7.4$ to $240 \mathrm{~nm}$. One unit of CAT is defined as the amount of enzyme which induces reduction in absorbance of 0.43 at $25^{\circ} \mathrm{C}$ for 3 minutes. The CAT activity was also expressed as units per milligram of protein (U/mg protein).

Reduced glutathione (GSH) and oxidized glutathione (GSSG) in the liver tissue were estimated using the method proposed by Vats et al. (36). Fifty $\mathrm{ml}$ of the homogenates of liver tissue was treated with $450 \mathrm{ml}$ solution of $10 \% \mathrm{~m}$-phosphoric acid and centrifuged for $10 \mathrm{~min}$ at $1000 \mathrm{x}$ g. For GSH determination, $0.1 \mathrm{ml}$ supernatant was used diluted with $1.8 \mathrm{ml}$ of $0.1 \mathrm{M}$ phosphate buffer ( $\mathrm{pH} 8$ ) containing $5 \mathrm{nmol} / \mathrm{L}$ EDTA, to which $0.1 \mathrm{ml}$ of o-phthalaldehyde in methanol $(1 \mathrm{mg} / \mathrm{ml})$ was added. After $15 \mathrm{~min}$ incubation, the emitted fluorescence was measured at $420 \mathrm{~nm}$ with an excitation of $350 \mathrm{~nm}$. The GSH level was expressed in nanomole per milligram of protein ( $\mathrm{nmol} / \mathrm{mg}$ protein).

The GSSG was estimated in $250 \mathrm{ml}$ supernatant which was incubated for 30 minutes with $40 \mathrm{nmol} / \mathrm{L}$ N-ethylmaleimide. Afterwards, $0.65 \mathrm{ml}$ of $0.1 \mathrm{~N} \mathrm{NaOH}$ was added. Further on, the same procedure was applied as with GSH but instead of phosphate buffer, $1.8 \mathrm{ml} 0.1$ $\mathrm{N} \mathrm{NaOH}$ and $0.1 \mathrm{ml}$ of o-phthalaldehyde were added to the $0.1 \mathrm{ml}$ reaction mixture.

The calculation of the GSH and GSSG concentrations was based on standard curves obtained with known concentrations of GSH and GSSG, respectively, which were processed in the same way.

\section{Histopathological study}

For the histological study, the hepatic samples were fixed in $10 \%$ buffered neutral formalin, embedded in paraffin, while sections were made at 4 micrometers and the slides were stained with hematoxylin and eosin (H\&E) dye. Then the slides were examined under an Olympus BX 51 microscope. The images were taken with an Olympus UC 30 digital camera and processed by a special image acquisition and processing program, i.e. Olympus Stream Basic.

\section{Statistical analysis}

The statistical analysis was performed using the SPSS software package (version 17.0). Data were reported as mean \pm SEM. One-way analysis of variance (ANOVA) was used to compare differences between groups, and two-way ANOVA for repeated measurements, followed by Tukey's multiple post-test comparisons, to compare the responses to Quercetin and exercise. Three-way ANOVA was used to compare cumulative response to Quercetin and exercise in diabetes. Differences were considered significant if $\mathrm{P}<0.05$. 


\section{Results}

Effects of Quercetin and Chronic Moderate Exercise on fasting blood glucose levels and body weight of normal and diabetic rats

Fig. 1. shows the fasting blood glucose (FBG) levels of all the experimental animals measured at the start of the experiment (first day), after 4 days ( $4^{\text {th }}$ day) and 7 days $\left(7^{\text {th }}\right.$ day) after the administration of STZ, and at the end of the experiment ( $35^{\text {th }}$ day).

The FBG was found to be significantly increased $(\mathrm{P}<0.05)$ in the diabetic groups (DS, DE, DSQ and DEQ) on the $4^{\text {th }}$ day and $7^{\text {th }}$ day when compared to the control groups confirming the onset of diabetes. The diabetic rats treated for 4 weeks with Que (DSQ group) showed a significant decrease $(\mathrm{P}<0.05)$ in the FBG levels suggesting its ameliorating role in diabetes. The chronic moderate regular exercise for 4 weeks significantly decreased $(\mathrm{P}<0.05)$ the FBG levels in the DE group when compared to the DS group while Que administration associated with chronic moderate regular exercise for 4 weeks (DEQ group) significantly decreased $(\mathrm{P}<0.0001)$ the FBG levels in diabetic rats when compared to the control diabetic rats (DS, DE and DSQ groups) (Fig. 1).

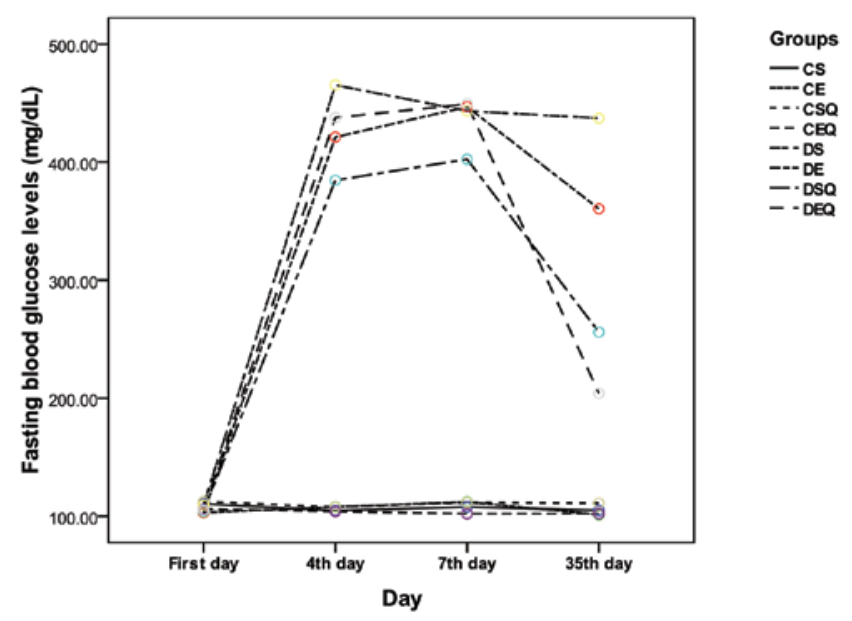

Fig. 1. The effects of Quercetin and chronic moderate exercise (training) on fasting blood glucose levels $(\mathrm{mg} / \mathrm{mg} \mathrm{dL})$ of control and diabetic rats. Results are the means \pm SD for ten animals each group.

In CS: non-diabetic sedentary untreated control rats, CE: non-diabetic trained untreated control rats, CSQ: non-diabetic sedentary control rats treated with Quercetin, CEQ: non-diabetic trained control rats treated with Quercetin, DS: diabetic sedentary control rats, DE: diabetic trained untreated control rats, DSQ: diabetic sedentary rats treated with Quercetin, DEQ: diabetic trained rats treated with Quercetin. Statistically significant differences are indicated by the symbols:

$\left({ }^{a}\right)$ vs. the sedentary untreated control rats $(\mathrm{CS}),\left({ }^{b}\right)$ vs. the sedentary diabetic untreated control rats (DS), $\left({ }^{c}\right)$ vs. diabetic, trained untreated control rats (DE) and $\left({ }^{d}\right)$ vs. diabetic, sedentary rats treated with Quercetin (DSQ). Means in a row with superscripts without a common letter differ, $\mathrm{P}<0.05$.

The body weight of all experimental rats is shown in Table I. At the end of the experiment ( $35^{\text {th }}$ day) diabetic rats (DS group) presented body weight loss. Diabetic rats treated for 4 weeks with Que (DSQ group) did not present significant differences $(\mathrm{P}>0.05)$ in body weight when compared to the sedentary untreated diabetic rats (DS group) (Table I). The 
chronic moderate regular exercise did not determine a significant decrease $(\mathrm{P}>0.05)$ in body weight in diabetic rats (DE group). The diabetic rats treated with Quercetin and subjected to chronic moderate regular exercise (DEQ group) did not show significant differences $(\mathrm{P}>$ 0.05 ) between the final and initial body weights (Table I).

Table 1. Effect of Quercetin and chronic moderate regular exercise (training) on body weight in normal and diabetic rats

\begin{tabular}{|l|c|c|c|c|c|c|c|c|}
\hline & CS & CE & CSQ & CEQ & DS & DE & DSQ & DEQ \\
\hline Initial body & $308.2 \pm$ & $305.2 \pm$ & $309.1 \pm$ & $306.1 \pm$ & $304.6 \pm$ & $307.3 \pm$ & $306.6 \pm$ & $308.8 \pm$ \\
weight $(\mathrm{g})$ & $5.73^{\mathrm{a}}$ & $3,29^{\mathrm{a}}$ & $5.15^{\mathrm{a}}$ & $7.42^{\mathrm{a}}$ & $9.14^{\mathrm{a}}$ & $3.71^{\mathrm{a}}$ & $3.43^{\mathrm{a}}$ & $3.39^{\mathrm{a}}$ \\
Final body & $310.8 \pm$ & $303.6 \pm$ & $310.2 \pm$ & $307.6 \pm$ & $279.4 \pm$ & $301.8 \pm$ & $285.3 \pm$ & $300.6 \pm$ \\
weight $(\mathrm{g})$ & $6.01^{\mathrm{a}}$ & $6.01^{\mathrm{a}}$ & $4.54^{\mathrm{a}}$ & $5.42^{\mathrm{a}}$ & $11.50^{\mathrm{b}}$ & $4.02^{\mathrm{a}}$ & $8.26^{\mathrm{b}}$ & $6.67^{\mathrm{a}}$ \\
\hline
\end{tabular}

Abbreviations: $\mathrm{CS}=$ control + sedentary, $\mathrm{CE}=$ control + exercise, $\mathrm{CEQ}=$ control + exercise + Quercetin, $\mathrm{DS}=$ diabetes + sedentary, $\mathrm{DE}=$ diabetes + exercise, $\mathrm{DSQ}=$ diabetes + sedentary + Quercetin, $\mathrm{DEQ}=$ diabetes + exercise + Quercetin.

Legend: Results are mean \pm SEM of 10 rats per each group. Statistically significant differences are indicated by the symbols: ${ }^{(a)}$ insignificant difference $(\mathrm{P}>0.05)$ between all experimental groups ${ }^{(*)}$ significant difference $(\mathrm{P}>0.05)$ between treated, trained and control groups.

Effects of Quercetin and Chronic Moderate Exercise on lipid peroxidation and proteins carbonylation in hepatic tissue homogenate of normal and diabetic rats

The MDA level has been widely used to determine lipid peroxidation in the tissue in experimental and clinical studies. Figure 2 shows the levels of MDA in the hepatic tissue homogenates of normal and diabetic rats after Que administration and chronic moderate exercise. The level of MDA (Fig. 2) in the liver significantly elevated $(\mathrm{P}<0.0001)$ after the induction of diabetes (DS group) as compared to the non-diabetic control sedentary rats (CS group). Diabetic rats when subjected to chronic moderate exercise (DE group) for 4 weeks exhibited non-significantly increased (P >0.05) MDA levels (Fig. 2) in the liver as compared to the control rats (DS group). Administration of Que to sedentary diabetic rats (DSQ group) for 4 weeks improved the significantly decreased $(\mathrm{P}<0.05)$ MDA levels (Fig. 2) in the liver as compared to the control rats (DS and DE groups). Diabetic rats treated with Que and subjected to chronic moderate exercise (DEQ group) for 4 weeks exhibited a significant decrease $(\mathrm{P}<0.05)$ in this marker of oxidative stress in the liver as compared to the control diabetic rats (DS and DE groups) (Fig. 2).

The effect of hyperglycemia on protein oxidation was measured by determining protein carbonyl (PC) groups in the hepatic tissue homogenate. Levels of PC in normal and diabetic rats after Que administration and chronic moderate exercise are shown in Fig. 3. PC levels in the liver significantly increased $(\mathrm{P}<0.0001)$ after the induction of diabetes (DS group) as compared to the non-diabetic sedentary control rats (CS group) (Fig. 3). When subjected to chronic moderate exercise (DE group) for 4 weeks, diabetic rats exhibited significantly decreased $(\mathrm{P}<0.05) \mathrm{PC}$ levels in the liver as compared to the control rats (DS group) (Fig. 3). The treatment of sedentary diabetic rats with Que (DSQ group) for 4 weeks significantly decreased $(\mathrm{P}<0.05)$ the $\mathrm{PC}$ levels in the liver as compared to the control rats (DS group) (Fig. 3). Diabetic rats treated with Que and subjected to chronic moderate exercise (DEQ group) for 4 weeks exhibited significantly decreased $(\mathrm{P}<0.05) \mathrm{PC}$ levels in the liver as compared to the control diabetic rats (DS, DE and DSQ groups) (Fig. 3). Que and chronic moderate exercise had cumulative effects $(\mathrm{P}<0.05)$ on the $\mathrm{PC}$ level decrease in the liver in diabetic rats. 


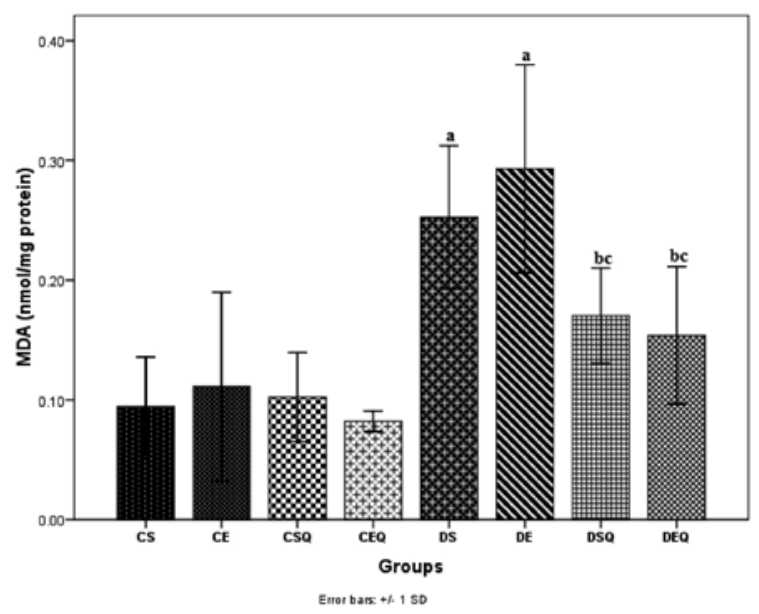

Fig. 2. The effects of Quercetin and chronic moderate exercise (training) on lipid peroxidation (MDA) (nmol/mg protein) levels in the hepatic tissue homogenates of control and diabetic rats. Results are the means $\pm \mathrm{SD}$ for ten animals each group. In CS: non-diabetic sedentary untreated control rats, CE: non-diabetic trained untreated control rats, CSQ: non-diabetic sedentary control rats treated with Quercetin, CEQ: non-diabetic trained control rats treated with Quercetin, DS: diabetic sedentary control rats, DE: diabetic trained untreated control rats,

DSQ: diabetic sedentary rats treated with Quercetin, DEQ: diabetic trained rats treated with Quercetin. Statistically significant differences are indicated by the symbols: ${ }^{\text {(a) }}$ vs. the sedentary untreated control rats (CS), ${ }^{\text {(b) }}$ vs. the sedentary diabetic untreated control rats (DS), ${ }^{(c)}$ vs. diabetic, trained untreated control rats (DE) and ${ }^{(d)}$ vs. diabetic, sedentary rats treated with Quercetin (DSQ).

Means in a row with superscripts without a common letter differ, $\mathrm{P}<0.05$.

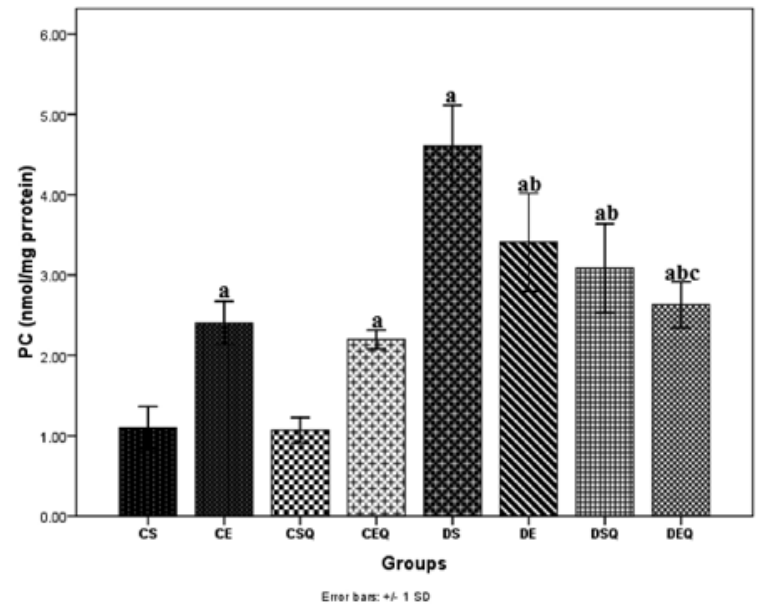

Fig. 3. The effects of Quercetin and chronic moderate exercise (training) on protein carbonyl groups (PC) (nmol mg protein) in the hepatic tissue homogenate of control and diabetic rats. Results are the means \pm SD for ten animals each group. In CS: non-diabetic sedentary untreated control rats, CE: non-diabetic trained untreated control rats, CSQ: non-diabetic sedentary control rats treated with Quercetin, CEQ: non-diabetic trained control rats treated with Quercetin, DS: diabetic sedentary control rats, DE: diabetic trained untreated control rats,

DSQ: diabetic sedentary rats treated with Quercetin, DEQ: diabetic trained rats treated with Quercetin. Statistically significant differences are indicated by the symbols: ${ }^{(a)}$ vs. the sedentary untreated control rats (CS),

${ }^{(b)}$ vs. the sedentary diabetic untreated control rats (DS), ${ }^{(c)}$ vs. diabetic, trained untreated control rats (DE) and ${ }^{(d)}$ vs. diabetic, sedentary rats treated with Quercetin (DSQ).

Means in a row with superscripts without a common letter differ, $\mathrm{P}<0.05$. 
Effects of Quercetin and Chronic Moderate Exercise on non-enzymatic antioxidants in hepatic tissue homogenate of normal and diabetic rats

The levels of reduced glutathione (GSH), a non-enzymatic antioxidant, and the GSH/ GSSG ratio in hepatic tissue homogenates of normal and diabetic groups is shown in Fig. 4. The GSH level (Fig. 4a) and the GSH/GSSG ratio (Fig. 4b) in the liver of diabetic sedentary rats (DS group) were found significantly decreased $(\mathrm{P}<0.05)$ as compared to the non-diabetic control sedentary rats (CS group). Quercetin administration significantly increased $(\mathrm{P}<0.05)$ the GSH level and the GSH/GSSG ratio in the liver of diabetic rats (DSQ group) as compared to the control rats (DS and DE group) (Fig. 4). Chronic moderate exercise decreased (P > 0.05 ) the GSH level and GSH/GSSG ratio in the liver of diabetic rats (DE group) in a nonsignificant way, but Que administration associated with chronic moderate exercise for 4 weeks significantly increased $(\mathrm{P}<0.05)$ the GSH level and the GSH/GSSG ratio in the liver of diabetic rats (DEQ group) as compared to the diabetic control rats (DS and DE groups) (Fig. 4).

a)

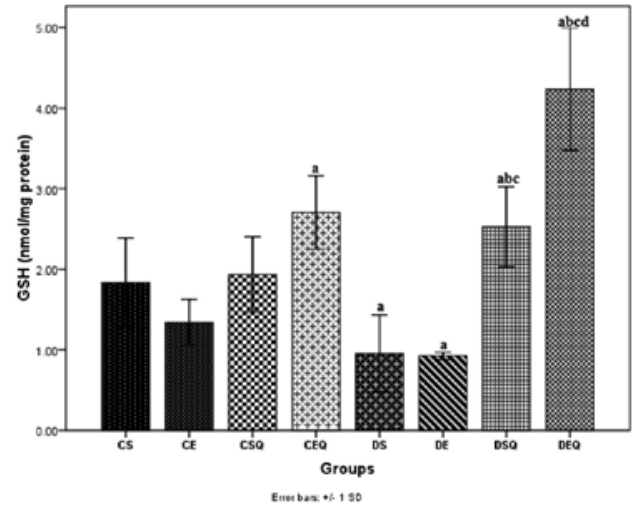

b)

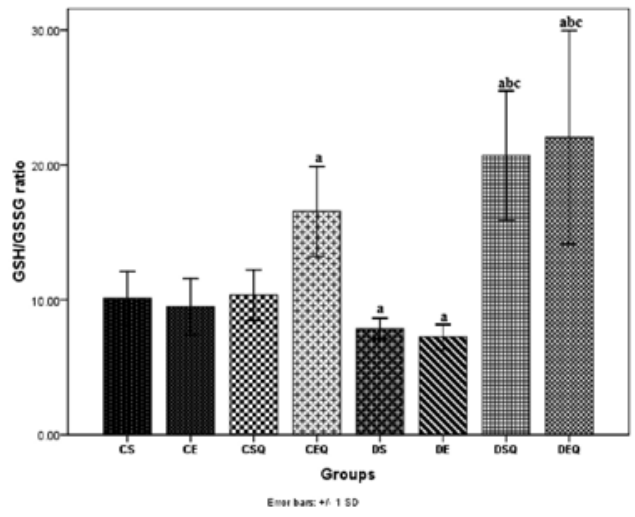

Fig. 4. The effects of Quercetin and chronic moderate exercise (training) on: (a) reduced glutathione (GSH) (nmol/mg protein) level and (b) GSH/GSSSG ratio in the hepatic tissue homogenate of control and diabetic rats.

Results are the means \pm SD for ten animals each group. In CS: non-diabetic sedentary untreated control rats, CE: non-diabetic trained untreated control rats, CSQ: non-diabetic sedentary control rats treated with Quercetin, CEQ: non-diabetic trained control rats treated with Quercetin, DS: diabetic sedentary control rats,

DE: diabetic trained untreated control rats, DSQ: diabetic sedentary rats treated with Quercetin, DEQ: diabetic trained rats treated with Quercetin. Statistically significant differences are indicated by the symbols:

${ }^{(a)}$ vs. the sedentary untreated control rats (CS), ${ }^{\text {(b) }}$ vs. the sedentary diabetic untreated control rats (DS), ${ }^{(c)}$ vs. diabetic, trained untreated control rats (DE) and ${ }^{(\mathrm{d})}$ vs. diabetic, sedentary rats treated with Quercetin (DSQ). Means in a row with superscripts without a common letter differ, $\mathrm{P}<0.05$.

Effects of Quercetin and Chronic Moderate Exercise on enzymatic antioxidant activity in hepatic tissue homogenate of normal and diabetic rats

The activities of enzymatic antioxidants (SOD and CAT) in the hepatic tissue homogenate were measured in normal and diabetic groups (Figs 5 and 6). In diabetic sedentary rats (DS group), the activities of SOD (Fig. 5) and CAT (Fig. 6) were significantly decreased (P < $0.0001)$ in the liver as compared to the CS group. The diabetic sedentary rats treated with Que (DSQ group) exhibited a significant increase $(\mathrm{P}<0.05)$ in the activities of these antioxidant enzymes in the liver as compared to diabetic control rats (DS group). Trained untreated diabetic control rats (DE group) also exhibited a significant increase $(\mathrm{P}<0.05)$ in the activities 
of these antioxidant enzymes in the liver as compared to the DS group. The diabetic rats treated with Que and subjected to chronic moderate exercise (DEQ group) exhibited a significantly increased $(\mathrm{P}<0.05)$ activity of SOD (Fig. 5) and CAT (Fig. 6) in the liver as compared to the diabetic control rats (DS, DE and DSQ groups). Que and the chronic moderate exercise had cumulative effects $(\mathrm{P}<0.05)$ on the increase of antioxidant enzymes (SOD and CAT) in the liver of diabetic rats.

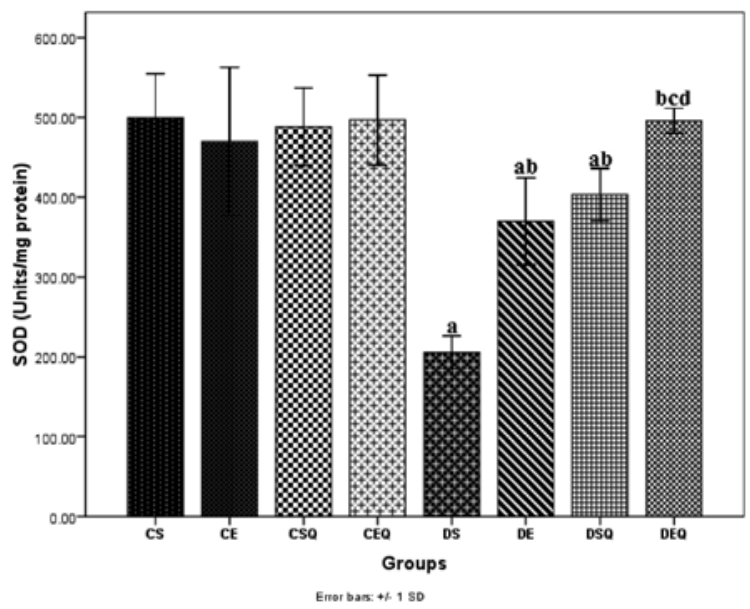

Fig. 5. The effects of Quercetin and chronic moderate exercise (training) on the level of superoxide dismutase (SOD) (Units/mg protein) activity in the hepatic tissue homogenate of control and diabetic rats. Results are the means \pm SD for ten animals each group. In CS: non-diabetic sedentary untreated control rats, CE: non-diabetic trained untreated control rats, CSQ: non-diabetic sedentary control rats treated with Quercetin, CEQ: non-diabetic trained control rats treated with Quercetin, DS: diabetic sedentary control rats, DE: diabetic trained untreated control rats, DSQ: diabetic sedentary rats treated with Quercetin, DEQ: diabetic trained rats treated with Quercetin. Statistically significant differences are indicated by the symbols: ${ }^{(a)}$ vs. the sedentary untreated control rats (CS),

(b) Vs. the sedentary diabetic untreated control rats (DS), ${ }^{(c)}$ vs. diabetic, trained untreated control rats (DE) and (d) vs. diabetic, sedentary rats treated with Quercetin (DSQ). Means in a row with superscripts without a common letter differ, $\mathrm{P}<0.05$.

Histopathological observations of the liver in normal and diabetic rats after Quercetin and Chronic Moderate Exercise

Figure 7 shows the section of liver tissue of all the experimental groups revealing the hepatocyte arrangement, central vein and portal tract. The examined sections showed normal liver architecture in the control groups (CS, CE, CSQ and CEQ). The hepatic tissue of diabetic rats (DS group) exhibiting mild periportal inflammatory cell infiltrate, vacuolization of the hepatocytes in the central and midlobular areas were visualized; these cells presented finely granular cytoplasm and relatively small vacuoles, without nuclear changes (Fig. 7E). The DE and DSQ groups showed normal liver architecture (Figs 7F and 7G). In the sections from the animals of DEQ group an increased number of Kuppfer cells were observed with no other morphological changes (Fig. 7H). 


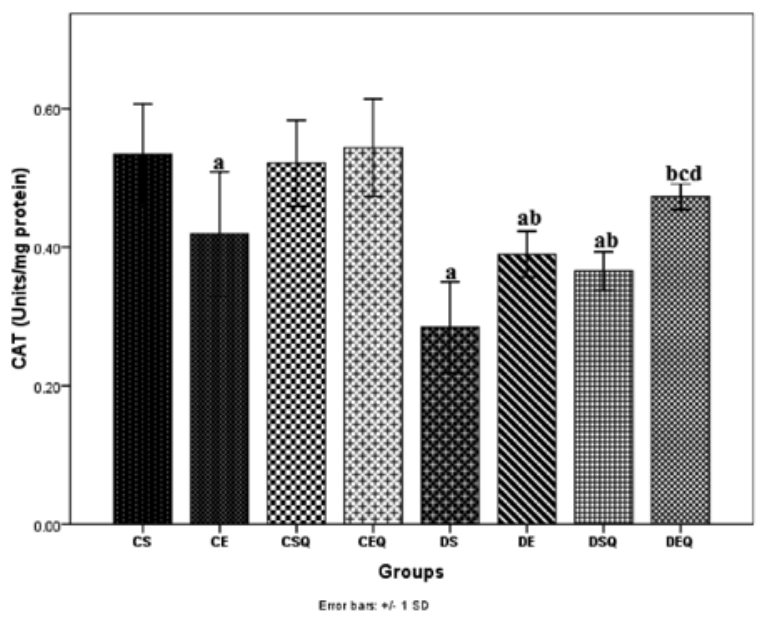

Fig. 6. The effects of Quercetin and chronic moderate exercise (training) on the level of catalse (CAT)

(Units/mg protein) activity in the hepatic tissue homogenate of control and diabetic rats.

Results are the means \pm SD for ten animals each group. In CS: non-diabetic sedentary untreated control rats, CE: non-diabetic trained untreated control rats, CSQ: non-diabetic sedentary control rats treated with Quercetin,

CEQ: non-diabetic trained control rats treated with Quercetin, DS: diabetic sedentary control rats,

DE: diabetic trained untreated control rats, DSQ: diabetic sedentary rats treated with Quercetin, DEQ: diabetic trained rats treated with Quercetin.

Statistically significant differences are indicated by the symbols: ${ }^{(a)}$ vs. the sedentary untreated control rats (CS),

(b) vs. the sedentary diabetic untreated control rats (DS), ${ }^{(c)}$ vs. diabetic, trained untreated control rats (DE) and ${ }^{(d)}$ vs. diabetic, sedentary rats treated with Quercetin (DSQ).

Means in a row with superscripts without a common letter differ, $\mathrm{P}<0.05$.
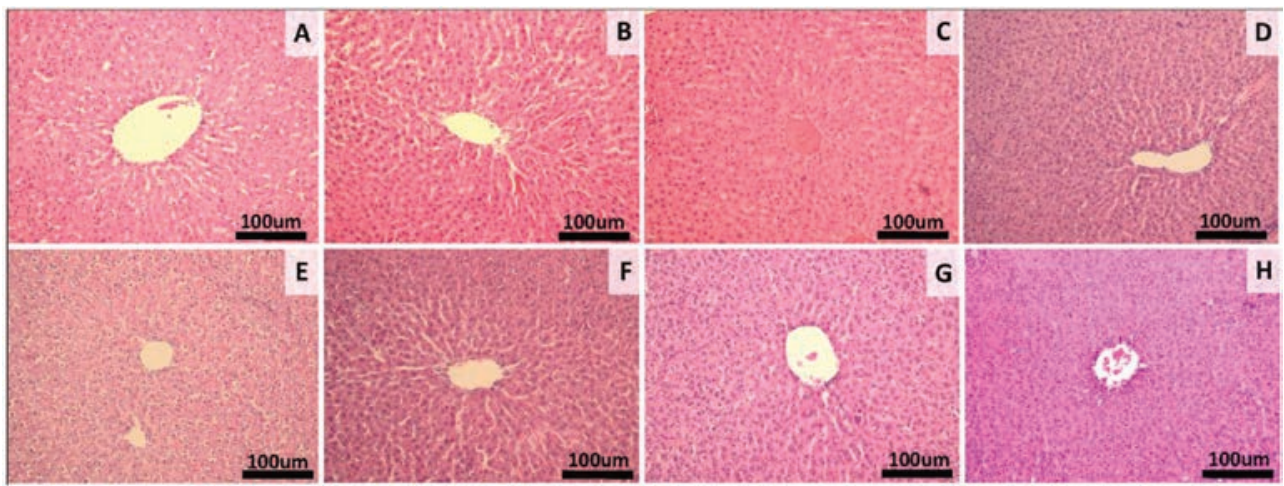

Fig. 7. Photomicrographs of hepatic tissue sections from all the experimental groups. Normal liver architecture, central area in the CS group; non-diabetic sedentary untreated control rats (A), CE group; non-diabetic trained untreated control rats (B), CSQ group; non-diabetic sedentary control rats treated with Quercetin (C), CEQ group; non-diabetic trained control rats treated with Quercetin (D), DE group; diabetic trained untreated control rats $(\mathrm{F})$ and DSQ group; diabetic sedentary rats treated with Quercetin (G). Mild periportal inflammatory cell infiltrate and

mild vacuolization of hepatocytes from the central area, without nuclear changes in the DS group: diabetic sedentary control rats (E); slight increase in the number of activated Kupffer cells in the DEQ group; diabetic trained rats treated with Quercetin $(H)$. H\&E stain, original magnification of $200 \times$. 


\section{Discussion}

The administration of streptozotocin (an N-nitroso derivative of glucosamine) induced diabetes in rats. Hyperglycemia induced by STZ can be similar to both types of diabetes mellitus (DM). The type of induced DM depends on: the dose of STZ, animal species, age of animal, animal weight, route of administration and nutritional status (12). The diabetogenic action of STZ is caused primarily by its specific cytotoxic action on the $\beta$-cells of the islets of Langerhans and secondly by their irreversible necrosis. The selective toxicity of the STZ upon the pancreatic $\beta$-cells was explained by the glucose moiety in its chemical structure that allows STZ to penetrate these cells by means of glucose transporter 2 (GLUT 2), which is present in the plasma membrane.

Other cells that express GLUT 2 transporter, such as the hepatocytes and the renal tubular cells are also susceptible to STZ, explaining why the experimental animals with STZinduced diabetes have renal and liver damage (12). In our present study we induced type 1 diabetes in rats with STZ. Ninety-six hours (4 days) after STZ administration, fasting blood glucose (FBG) levels were significantly elevated in the diabetic rats in our study.

In the present study we have shown that Quercetin works as an antidiabetic agent by the reduction of FBG levels in the diabetic sedentary rats. Our results are consistent with recent research that revealed the hypoglycemic effect of Quercetin in animal models of type 1 diabetes $(1,2,19,22)$. Quercetin is a polyphenol of the flavonol group known for its many beneficial effects on the body. The Quercetin mechanism that lowers blood sugar was explained by: its $\alpha$-glycosidase inhibitory activity in vitro; the increase of the hexokinase activity; the decrease in the glucose-6-phosphatase and fructo-bisphosphatase activities; the increased expression of GLUT 4 (insulin-dependent glucose transporter) via mRNA expression and translocation to the plasma membrane (1).

Diabetic rats subjected to chronic moderate regular exercise for four weeks in our study showed significantly decreased levels of FBG when compared to the sedentary diabetic rats. In the current study we chose swimming as a model of physical effort because it is a method that causes less mechanical stress and injury and produces a redistribution of the blood flow to the tissues without significant variations in the heart rate and cardiac output, minimizing the intensity of injuries caused by the generation of $\operatorname{ROS}(9,23,31,35)$. Our results, consistent with recent studies, showed that moderate swimming training decreased glycemia in diabetic rats $(3,15)$.

In the present research, Quercetin administration associated with chronic moderate regular exercise for 4 weeks in diabetic rats (DEQ group) significantly decreased the FBG levels in diabetic rats when compared to the control groups. This positive change in the glycemic level might be explained by multiple mechanisms. Several studies reported that physical exercise in diabetes enhances insulin sensitivity, protects $\beta$-cells of Langerhans islets (9), causes structural and functional changes in the skeletal muscle, which involves an increase in glucose transport and in glycogen synthesis, etc. $(3,9,33)$.

Quercetin administration associated with moderate swimming training have synergistic hypoglycemic effects because both protect the $\beta$-cells of Langerhans islet and increase insulin release and the GLUT-4 expression, thus increasing the intracellular glucose transport by insulin-independent pathways $(1,2,9,33)$.

STZ-induced diabetic rats showed clinical signs that were specific of severe DM: weight loss, polyuria, polydipsia and polyphagia associated with hyperglycemia $(28,34)$. In the present study sedentary diabetic rats (DS group) showed body weight loss and the training protocol restored this body weight reduction caused by the diabetic state. Quercetin 
administration in diabetic rats had no effect on diabetes-induced body weight decrease but Quercetin administration associated with moderate swimming training for 4 weeks prevented body weight reduction caused by the diabetic condition.

Diabetes mellitus is a metabolic disease characterized by hyperglycemia resulting from defective insulin secretion or resistance insulin action. Hyperglycemia increases the production of ROS through protein glycation. Auto-oxidation of unsaturated lipids in plasma and membrane lipids is also responsible for generating free radicals. The produced free radicals react with polyunsaturated fatty acids in the cell membrane leading to lipid peroxidation. Oxidative stress is responsible for the pancreatic $\beta$-cell dysfunction caused by glucose toxicity in hyperglycemia (12). The STZ-induced diabetes model exhibits high oxidative stress due to persistent and chronic hyperglycemia, decreasing the activity of the antioxidative defense system and thus promoting generation of free radicals (12). The free radicals lead to glycation reactions and oxidative modification of lipids, protein modification, and DNA damage. Our results are inkeeping with other recent studies that state the fact that diabetes mellitus increases oxidative stress in the liver in an animal model system $(1,30)$. The present study found a significant increase in the levels of MDA, which is an end product of lipoperoxidation, and PC, produced by protein oxidation, associating with lowering antioxidant activities in the liver which demonstrates the presence of oxidative stress in STZinduced diabetic rats, at this level.

Quercetin has been shown to be a potent flavonoid that acts as a potent antidiabetic agent through its anti-inflammatory and antioxidant effects $(1,2,11)$. The results of the present study also show a decrease in the levels of MDA and PC in the liver of diabetic rats after Quercetin supplementation. These results were found to be inkeeping with recent findings reported by other authors $(2,11)$.

The present study suggests that Quercetin improves liver oxidative damage by scavenging chronic hyperglycemia-induced free radicals. Enzymatic (SOD and CAT) and non-enzymatic (GSH, GSH/GSSG ratio) endogenous antioxidants are responsible for the neutralisation of the ROS. It is known that STZ-treated $\beta$-cells generate oxygen free radicals (12) and ROS may inactivate and reduce SOD, CAT, GPx and GST activities in the liver and the antioxidant enzymes play an important role in protecting the hepatic tissue from oxidative damage (30). In the present study the low levels of activity of SOD and CAT in the liver of diabetic rats indicated diabetes-induced stress and a significant elevation of these antioxidants was observed in the liver of Quercetin -treated diabetic rats, which was due to its scavenging property.

GSH functions as free radical scavenger and recent studies showed that the tissue GSH concentrations in STZ-induced diabetic rats were significantly lower, this non-enzymatic antioxidant functioning as a direct free radical scavenger, cofactor for many enzymes; it is also involved in the maintenance of exogenous antioxidants such as vitamins $\mathrm{E}$ and $\mathrm{C}$ in their active sites $(13,16,30)$. In the present study, decreased levels of GSH and GSH/GSSG ratio were observed in the liver of diabetic rats; whereas Quercetin-treated diabetic rats showed a significant elevation of these antioxidants in the liver. This could be due to the ability of Quercetin to directly scavenge ROS and free radicals, chelate metal catalysts and activate antioxidant enzymes $(6,19)$.

Exercise training has been known to be effective in diabetes by increasing insulin sensitivity, decreasing of oxidative stress and preserving the pancreatic $\beta$-cell integrity (9). Swimming protocols were used in another studied issue in order to evaluate the effects of exercise training on the diabetes pathophysiology, inflammation and oxidative stress, included 
$(3,9,23,35)$. In the present research study, moderate chronic exercise (training) reduced oxidative stress in the liver of diabetic rats and Quercetin administration associated with chronic moderate exercise significantly restored the elevated MDA and PC levels and increased the antioxidant activities in the liver of STZ-induced diabetic rats. In our study the treatment with Quercetin increased the activities of SOD and CAT; it increased the GSH level and GSH/GSSG ratio in the liver of STZ-induced diabetic rats but Quercetin supplementation associated with chronic moderate exercise significantly increased antioxidant capacity of the liver.

In the present research we studied the histological changes in the liver in diabetic sedentary rats, diabetic trained rats, diabetic sedentary rats treated with Quercetin $(20 \mathrm{mg} / \mathrm{kg}$ body weight) and diabetic trained rats also treated with Quercetin in order to assess the preservative role in these tissues. Diabetic liver shows mild periportal inflammatory cell infiltrate, vacuolization of the hepatocytes was seen in the central and midlobular areas, these cells presenting finely granular cytoplasm and relatively small vacuoles, without nuclear changes. Diabetic trained rats and diabetic sedentary rats also treated with Quercetin showed normal liver architecture. Quercetin-treated diabetic trained rats revealed normal liver architecture with an increased number of Kuppfer cells.

These results suggest that the administration of Quercetin associated with moderate exercise training exerts synergistic effects in protecting the liver in diabetes, possibly due to the significant attenuation of hyperglycemia and hyperglycemia-mediated oxidative stress by decreasing lipid peroxidation and protein oxidation and preserving the structural and functional integrity of the liver tissues.

\section{Conclusion}

In conclusion, our findings suggest that Quercetin administration in association with chronic moderate exercise (training) exerts protective effects in diabetes by decreasing hyperglycemiamediated oxidative stress and histopathological changes in the liver tissue.

\section{Acknowledgements}

This research was supported under the contract funded by "Iuliu Hatieganu” University of Medicine and Pharmacy, Cluj-Napoca, Romania, through the internal grant numbered 1494/8/28.01.2014. We gratefully thank Mr Remus Moldovan for animal handling. We also thank Nicoleta Decea for her help regarding oxidative stress parameters' assessment.

\section{REFERENCES}

1. Alam MM, Meerza D, Naseem I: Protective effect of quercetin on hyperglycemia, oxidative stress and DNA damage in alloxan induced type 2 diabetic mice. Alam Life Sci. 109, 8-14 (2014)

2. Arya A, Jamil Al-Obaidi MM, Shahid N, Bin Noordin MI, Looi CY, Wongd WF, Khaing SL, Mustafa MR: Synergistic effect of quercetin and quinic acid by alleviating structural degeneration in the liver, kidney and pancreas tissues of STZ-induced diabetic rats: A mechanistic study. Food Chem. Toxicol. 71, 183-196 (2014)

3. Arshadi S, Bakhtiyari S, Haghani K, Valizadeh A: Effects of Fenugreek seed extract and swimming endurance training on plasma glucose and cardiac antioxidant enzymes activity in streptozotocin-induced diabetic rats. Osong Public Health Res. Perspect. 6, 87-93 (2015)

4. Babujanarthanam R, Kavitha P, Mahadeva Rao US, Pandian MR: Quercitrin a bioflavonoid improves the antioxidant status in streptozotocin-induced diabetic rat tissues. Mol. Cell Biochem. 358, 121-129 (2011) 
5. Bakhshaeshi M, Khaki A, Fathiazad F, Khaki AA, Ghadamkheir E: Anti-oxidative role of quercetin derived from Allium cepa on aldehyde oxidase (OX-LDL) and hepatocytes apoptosis in streptozotocin-induced diabetic rat. Asian Pac. J. Trop. Biomed. 2, 528-531 (2012)

6. Boots AW, Haenen GR, Bast A: Health effects of quercetin: from antioxidant to nutraceutical. Eur. J. Pharmacol. 585, 325-337 (2008)

7. Chis IC, Ungureanu MI, Marton A, Simedrea R, Muresan A, Postesc, ID, Decea N: Antioxidant effects of a grape seed extract in a rat model of diabetes mellitus. Diab. Vasc. Dis. Res. 6, 200-204 (2009)

8. Conti M, Morand PC, Levillain P: Improved fluoromeric determination of malonaldehyde. Clin. Chem. 37, 1273-1275 (1991)

9. Coskun O, Ocakci A, Bayraktaroglu T, Kanter M: Exercise training prevents and protects streptozotocininduced oxidative stress and beta-cell damage in rat pancreas. Tohoku J. Exp. Med. 203, 145-54 (2004)

10. Di Naso FC, Simoes Dias A, Porawski M, Marroni NA: Exogenous superoxide dismutase: action on liver oxidative stress in animals with streptozotocin-induced diabetes. Exp. Diab. Res., Article ID 754132, 6 pages (2011)

11. Dias AS, Porawski M, Alonso M, Marroni N, Collado PS, González-Gallego J: Quercetin decreases oxidative stress, NF-kappaB activation, and iNOS over expression in liver of streptozotocin-induced diabetic rats. J. Nutr. 135, 2299-2304 (2005)

12. Eleazu CO, Eleazu KC, Chukwuma S, Essien UN: Review of the mechanism of cell death resulting from streptozotocin challenge in experimental animals, its practical use and potential risk to humans. J. Diabetes Metab. Disord. 12, 60 (2013)

13. Ewis SA, Abdel-Rahman MS: Effect of metformin on glutathione and magnesium in normal and streptozotocininduced diabetic rats. J. Appl. Toxicol. 15, 387-390 (1995)

14. Formagio ASN, Kassuya CAL, Neto FF, Volobuff CRF, Iriguchi EKK, Vieira M, Foglio MA: The flavonoid content and antiproliferative, hypoglycaemic, anti-inflammatory and free radical scavenging activities of Annona dioica St. Hill. BMC Complement. Altern. Med. 13, 14-22 (2013)

15. Gomes RJ, de Mello MA, Caetano FH, Sibuya CY, Anaruma CA, Rogatto GP, Pauli JR, Luciano E: Effects of swimming training on bone mass and the GH/IGF-1 axis in diabetic rats. Growth Horm. IGF Res. 16, 326-331 (2006)

16. Guoyao W. Fang ZY, Yag S, Lupton RJ, Turner DN: Glutathione metabolism and implications for health. J. Nutr. 134, 489-492 (2004)

17. Hsieh PS, Hsieh YJ: Impact of liver diseases on the development of type 2 diabetes mellitus. World J. Gastroenterol. 17, 5240-5245 (2011)

18. Inoguchi T, Li P, Umeda F, Yu HY, Kakimoto M, Imamura M, Aoki T, Etoh T, Hashimoto T, Naruse M, Sano H, Utsumi $\mathrm{H}$, Nawata $\mathrm{H}$ : High glucose level and free fatty acid stimulate reactive oxygen species production through protein kinase $\mathrm{C}$-dependent activation of $\mathrm{NAD}(\mathrm{P}) \mathrm{H}$ oxidase in cultured vascular cells. Diabetes 49, 1939-1945 (2000)

19. Jeong SM, Kang MJ, Choi HN, Kim JH, Kim JI: Quercetin ameliorates hyperglycemia and dyslipidemia and improves antioxidant status in type 2 diabetic db/db mice. Nutr. Res. Pract. 6, 201-207 (2012)

20. Kakkar P, Das B, Viswanthan PN: A modified spectrophotometric assay of superoxide dismutase (SOD). Indian J. Biochem. Biophys. 21, 130-132 (1984)

21. Kamalakkannan N, Stanely M, Prince P: The antihyperglycaemic and antioxidant effect of rutin, a polyphenolic flavonoid, in streptozotocin-induced diabetic Wistar rats. Basic Clin. Pharmacol. Toxicol. 98, 97-103 (2006)

22. Kanter M, Aktas C, Erboga M: Protective effects of quercetin against apoptosis and oxidative stress in streptozotocin-induced diabetic rat testis. Food Chem. Toxicol. 50, 719-725 (2012)

23. Lee S, Park Y, Dellsperger KC, Zhang C: Exercise training improves endothelial function via adiponectindependent and independent pathways in type 2 diabetic mice. Am. J. Physiol. Heart. Circ. Physiol. 301, 306314 (2011)

24. Lucchesi AN, Freitas NT, Cassettari LL, Marques SF, Spadella CT: Diabetes mellitus triggers oxidative stress in the liver of alloxan-treated rats: a mechanism for diabetic chronic liver disease. Acta Cir. Bras. 28, 502-508 (2013)

25. Moussa SA: Oxidative stress in diabetes mellitus. Romanian J. Biophys. 18, 225-236 (2008)

26. Mrowicka M: The role of disorders of the prooxidant-antioxidant system in diabetes etiopathology. Postepy. Hig. Med. Dosw. 65, 534-541 (2011)

27. Nagasawa T, Tabata N, Ito Y, Aiba Y, Nishizawa N, Kitts DD: Dietary G-rutin suppresses glycation in tissue proteins of streptozotocin-induced diabetic rats. Mol. Cell Biochem. 252, 141-147 (2003) 
28. Oelze M, Knorr M, Schuhmacher S, Heeren T, Otto C, Schulz E, Reifenberg K, Wenzel P, Münzel T, Daiber A: Vascular dysfunction in streptozotocin-induced experimental diabetes strictly depends on insulin deficiency. J. Vasc. Res. 48, 275-284 (2011)

29. Pippenger CE, Browne RW, Armstrong D (1998): Regulatory antioxidant enzymes. In: Methods in Molecular Biology, vol. 108: Free Radicals and Antioxidant Protocols D. Armstrong. Humana Press Inc. Totowa NJ. pp 299-309

30. Prabakaran D, Ashokkumar N: Protective effect of esculetin on hyperglycemia-mediated oxidative damage in the hepatic and renal tissues of experimental diabetic rats. Biochimie 95, 366-373 (2013)

31. Ravi Kiran T, Subramanyam MV, Asha Devi S: Swim exercise training and adaptations in the antioxidant defense system of myocardium of old rats: relationship to swim intensity and duration. Comp. Biochem. Physiol. B. Biochem. Mol. Biol. 137, 187-196 (2004)

32. Reznick AZ, Packer L: Oxidative damage to proteins: spectrophotometric method for carbonyl assay. Methods Enzymol. 233, 347-357 (1994)

33. Rocha RE, Coelho I, Pequito DC, Yamagushi A, Borghetti G, Yamazaki RK, Brito GA, Machado J, Kryczyk M, Nunes EA, Venera G, Fernandes LC: Interval training attenuates the metabolic disturbances in type 1 diabetes rat model. Arq. Bras. Endocrinol. Metabol. 57, 594-602 (2013)

34. Szkudelski T: The mechanism of alloxan and streptozotocin action of $\beta$-cells of the rat pancreas. Physiol. Res. 50, 537-546 (2001)

35. Teixeira de Lemos E, Pinto R, Oliveira J, Garrido P, Sereno J, Mascarenhas-Melo F, Pascoa-Pinheiro J, Teixeira F, Reis F: Differential effects of acute (extenuating) and chronic (training) exercise on inflammation and oxidative stress status in an animal model of type 2 diabetes mellitus. Mediators of Inflammation. Article ID 253061, 8 pages (2011)

36. Vats P, Singh VK, Singh SN, Singh SB: Glutathione metabolism under high-altitude stress and effect of antioxidant supplementation. Aviation, Space and Environmental Medicine. 79, 1106-1111 (2008)

37. Yang H, Jin X, Kei Lam CW, Yan SK: Oxidative stress and diabetes mellitus. Clin. Chem. Lab. Med. 49, 1773-1782 (2011)

38. Zhang H, Zhang C: Vasoprotection by dietary supplements and exercise: role of TNF $\alpha$ signaling. Exp. Diab. Res. Article ID 972679, 6 pages (2012) 